\title{
Outlier rejection for cameras on intelligent vehicles
}

\author{
Jae Kyu Suhr ${ }^{\text {a }}$, Ho Gi Jung ${ }^{\text {a,b }}$, Kwanghyuk Bae ${ }^{\text {a }}$, Jaihie Kim ${ }^{\text {a,* }}$ \\ ${ }^{a}$ School of Electrical and Electronic Engineering, Yonsei University, Seoul 120-749, South Korea \\ ${ }^{\mathrm{b}}$ Global R\&D H.Q., Mando Corporation, Yongin-Si 446-901, South Korea
}

Received 7 June 2007; received in revised form 20 September 2007

Available online 23 December 2007

Communicated by W. Zhao

\begin{abstract}
This paper proposes an algorithm for rejecting false matches (known as outliers) in image pairs acquired with automobile-mounted cameras. Many intelligent vehicle applications require point correspondences for motion estimation and 3D reconstruction which can be affected by outliers. We use the property of automobile motion to reject outliers. Automobile motion mostly represented by two translations and one rotation. The proposed algorithm eliminates the rotational effect and estimates the focus of expansion (FOE). Once the FOE is estimated, the joining line directions with respect to the FOE are used for rejecting the outliers. This algorithm is simple and its computational cost is independent of the outlier percentage. Experimental results show that the proposed algorithm rejects a large number of outliers but retains most inliers when working with synthetic and real image pairs. It works even when the initial matches are contaminated by $80-90 \%$ of the outliers. As a pre-processing step of RANSAC, it significantly reduces the computational cost when initial matches include a large number of outliers.
\end{abstract}

(C) 2007 Elsevier B.V. All rights reserved.

Keywords: Outlier rejection; Motion constraint; Focus of expansion; Automobile motion; Intelligent vehicle

\section{Introduction}

Recently, single moving cameras have been widely used in intelligent vehicle applications for 3D reconstruction (Mouragnon et al., 2006a; Suhr et al., 2007), SLAM (Mouragnon et al., 2006b; Royer et al., 2005), obstacle detection (Sun et al., 2006; Yamaguchi et al., 2006), and parking assistant systems (Fintzel et al., 2004; Jung et al., 2006; Vestri et al., 2005). Many of these applications rely on motion estimation and 3D reconstruction techniques, which require point correspondences between their image pairs (Ma and Soatto, 2000; Hartley and Zisserman, 2000). Therefore, matching the corresponding points is very important.

\footnotetext{
* Corresponding author.

E-mail addresses: 1fisbf@yonsei.ac.kr(J.K.Suhr),hgjung@mando.com (H.G. Jung), paero@yonsei.ac.kr (K. Bae), jhkim@yonsei.ac.kr (J. Kim).
}

There has been intensive research into finding corresponding points. This research can be divided into three groups. The first group uses a simple point detector and a simple image intensity similarity (Goshtasby, 2005; Schmid and Mohr, 2000; Smith and Brady, 1997). The second group uses an invariant point detector and an invariant local descriptor (Dufournaud et al., 2000; Lowe, 2004; Mikolajczyk and Schmid, 2004; Mikolajczyk and Schmid, 2005). The last group tracks feature points by using image sequences (Lucas and Kanade, 1981; Tomasi and Shi, 1994).

The first group can find corresponding points at a low computational cost, although many matching errors may occur. The second and last groups can find corresponding points reliably, but their computational costs are high because complex algorithms and many image sequences have to be used, respectively. If a first group method is used for real-time application due to its computational simplicity (Goshtasby, 2005; Vestri et al., 2005) or if multiple candidate matches are produced for each feature point (Zhang 
Table 1

Required RANSAC sampling numbers with respect to outlier percentages

\begin{tabular}{llllllllll}
\hline Outlier percentage & $10 \%$ & $20 \%$ & $30 \%$ & $40 \%$ & $50 \%$ & $60 \%$ & $70 \%$ & $80 \%$ & $90 \%$ \\
\hline Sampling number & 8 & 25 & 78 & 272 & 1177 & 7025 & 70,188 & $1,798,892$ & $460,517,014$ \\
\hline
\end{tabular}

and Kosecka, 2006), a large number of matching errors are likely to occur. These matching errors can be categorized into two types. The first type occurs if a match is missed and the second type occurs if a false match is found (Adam et al., 2001). We refer to the latter type as an outlier.

These outliers can severely affect motion estimation and $3 \mathrm{D}$ reconstruction, especially when the least-squares-based algorithm is used (Hartley, 1995). For this reason, robust estimation algorithms have been intensively researched in order to produce meaningful results in spite of the presence of outliers (Salvi et al., 2001; Torr and Murray, 1997; Torr and Zisserman, 1998; Zhang, 1998). However, the computational cost of many of these algorithms depends on the percentage of outliers. In the case of RANSAC (Fischler and Bolles, 1981), the expected sampling number dramatically increases with a higher outlier percentage. Table 1 shows the sampling number required to estimate the fundamental matrix using Hartley's 8-point algorithm with a $99 \%$ guarantee. This table is calculated by using the equation in Fischler and Bolles's paper (Fischler and Bolles, 1981). From Table 1, it is clear that RANSAC is impractical when the outlier percentage is large. Therefore, outlier rejection is inevitable when initial matches are contaminated by a large number of outliers.

There has been research in terms of rejecting outliers when initial matches include a large number of outliers (Adam et al., 2001; Grinstead and Koschan, 2006). Adam et al. (2001) proved that all the lines joining the correct matches make a common orientation when the feature points in one image are rotated by a certain angle. These researchers estimated the most appropriate angle and the common orientation using a mean shift mode estimator, and then rejected the matches whose joining lines did not follow the common orientation. This algorithm is effective for general camera motions. However, as mentioned in their paper, this algorithm can only deal with the image pairs taken by a narrow-angle camera. This limitation is critical for intelligent vehicle applications because many current applications adopt wide-angle cameras (Fintze1 et al., 2004; Gandhi and Trivedi, 2005; Jung et al., 2006; Mouragnon et al., 2006a,b; Royer et al., 2005; Suhr et al., 2007; Takahashi et al., 2002; Vestri et al., 2005).

Grinstead and Koschan (2006) found that the orientations of the lines joining the correct matches were the same when camera motion was perpendicular to its optical axis. They calculated the orientations of all the joining lines and estimated the probability distribution in order to find the common orientation. Once the common orientation was estimated, the matches whose joining lines did not follow that orientation were identified as outliers. However, this algorithm also presents a strict limitation, since the camera

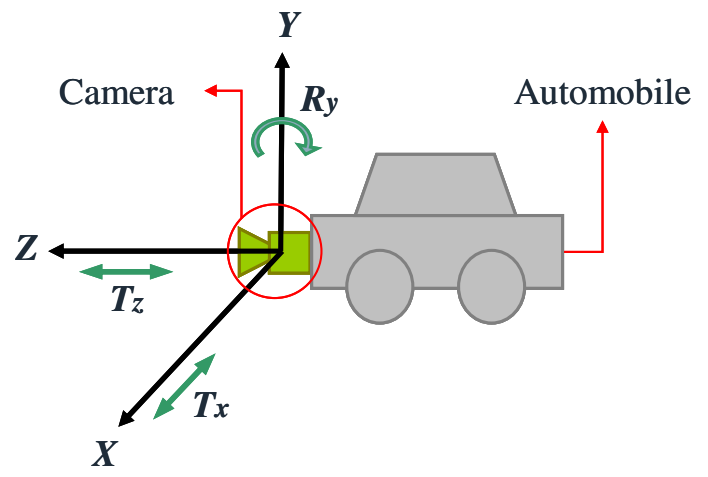

Fig. 1. Configuration of automobile rear view camera. Camera motion mostly consists of two translations $\left(T_{x}\right.$ and $\left.T_{z}\right)$ and one rotation $\left(R_{y}\right)$.

motion must be perpendicular to the optical axis of the camera. This kind of motion never occurs in automobile rear view cameras, as shown in Fig. 1. We used a general camera configuration in intelligent vehicle applications (Batavia et al., 1997; Burger and Bhanu, 1990; Fintze1 et al., 2004; Jung et al., 2006; Mouragnon et al., 2006a,b; Royer et al., 2005; Suhr et al., 2007; Takahashi et al., 2002; Vestri et al., 2005).

Because those two algorithms are not suitable for automobile rear view wide-angle cameras, we propose a new algorithm, which uses the property of automobile motion to reject outliers. Automobile motion mostly consists of two translations $\left(T_{x}\right.$ and $\left.T_{z}\right)$ and one rotation $\left(R_{y}\right)$ when we assume two situations. One situation is when the optical axis of the camera is parallel to the road and the other situation is when the surface of the road is flat. The first situation can be forced by using a pre-calibrated camera angle. The second situation occurs when the road between two different camera positions is reasonably flat or when the time interval between the two images is sufficiently small.

The proposed algorithm eliminates the $Y$-axis rotation $\left(R_{y}\right)$ and then estimates the focus of expansion (FOE). First, it transforms the feature points in one of two images by a homography to eliminate the $Y$-axis rotational effect. Then, the joining lines of the initial matches are accumulated and the peak value and its location are saved. This process is repeated with various angles within the search range. With the correct rotation angle, the rotation effect is totally eliminated by a homography and the accumulation contains the largest peak value at the location of the FOE. Therefore, the rotation angle causing the largest peak is the $Y$-axis rotation angle and its location is the FOE. Once the FOE is estimated, matches whose joining lines do not point inward or outward with respect to the FOE 
are identified as outliers. This algorithm is simple and its computational cost does not depend on the outlier percentage so it can be used as a pre-processing step of a robust estimation algorithm to lower computational costs.

In the experiments, the performance and robustness of the proposed algorithm were tested with synthetic and real image pairs contaminated by $10-90 \%$ of outliers. Using these image pairs, we compared the performance of Adam's algorithm and the proposed algorithm, and then compared the computational costs of RANSAC alone and RANSAC with the proposed algorithm. Experimental results show that the proposed algorithm rejected a large number of outliers but retained most of the inliers when using synthetic and real image pairs. With real image pairs, it rejected $86.5 \%$ of the outliers and retained $96.3 \%$ of the inliers. As a pre-processing step, it significantly reduced computation time.

The rest of this paper is organized as follows: in Section 2 , we discuss the motion field for general motion and automobile motion. In Section 3, we explain the algorithm for FOE estimation and outlier rejection. In Section 4, we show the experimental results and compare the results with previous algorithms. Finally, in Section 5, we conclude the paper with a summary and a future work.

\section{Motion field}

\subsection{Motion field for general motion}

Motion field refers to the 2D vector field of the velocities of image points, caused by the relative motion between a camera and an observed scene (Trucco and Verri, 1998). $\boldsymbol{P}=[X, Y, Z]^{\mathrm{T}}$ represents a $3 \mathrm{D}$ scene point in the camera reference frame. The camera center is the origin, the optical axis is the $Z$-axis, and $f$ denotes the focal length. The image of a scene point, $\boldsymbol{P}$, is the point $\boldsymbol{p}$ given by:

$\boldsymbol{p}=f \frac{\boldsymbol{P}}{Z}$

Since the third coordinate of $\boldsymbol{p}$ is equal to $f$, we write $\boldsymbol{p}=[x, y]^{\mathrm{T}}$ instead of $\boldsymbol{p}=[x, y, f]^{\mathrm{T}}$. The relative motion between $\boldsymbol{P}$ and the camera is then described as:

$\boldsymbol{V}=-\boldsymbol{T}-\boldsymbol{\omega} \times \boldsymbol{P}$

$\boldsymbol{T}$ represents the translational component of the motion, and $\omega$ represents the angular velocity. As the motion is rigid, $\boldsymbol{T}$ and $\boldsymbol{\omega}$ are the same for any $\boldsymbol{P}$. Broken into individual components, Eq. (2) reads:

$$
\begin{aligned}
& V_{x}=-T_{x}-\omega_{y} Z+\omega_{z} Y \\
& V_{y}=-T_{y}-\omega_{z} X+\omega_{x} Z \\
& V_{z}=-T_{z}-\omega_{x} Y+\omega_{y} X
\end{aligned}
$$

To obtain the relationship between the velocity of $\boldsymbol{P}$ in space and the corresponding velocity of $\boldsymbol{p}$ on the image plane, we take the time derivative of both sides of Eq.
(1), which produces motion field equations. The motion field, $\boldsymbol{v}$, is given by:

$\boldsymbol{v}=f \frac{Z \boldsymbol{V}-V_{z} \boldsymbol{P}}{Z^{2}}$

Broken into individual components, Eq. (4) reads:

$$
\begin{aligned}
& v_{x}=\frac{T_{z} x-T_{x} f}{Z}-\omega_{y} f+\omega_{z} y+\frac{\omega_{x} x y}{f}-\frac{\omega_{y} x^{2}}{f} \\
& v_{y}=\frac{T_{z} y-T_{y} f}{Z}+\omega_{x} f-\omega_{z} x-\frac{\omega_{y} x y}{f}+\frac{\omega_{x} y^{2}}{f}
\end{aligned}
$$

\subsection{Motion field for automobile motion}

Automobile motion mostly consists of two translations ( $T_{x}$ and $\left.T_{z}\right)$ and one rotation $\left(R_{y}\right)$, as shown in Fig. 1. In this case, Eq. (5) is simplified as:

$$
\begin{aligned}
& v_{x}=\frac{T_{z} x-T_{x} f}{Z}-\omega_{y} f-\frac{\omega_{y} x^{2}}{f} \\
& v_{y}=\frac{T_{z} y}{Z}-\frac{\omega_{y} x y}{f}
\end{aligned}
$$

Eq. (6) is divided into the translational component and the rotational component. These components are denoted as $\boldsymbol{v}_{t}$ and $\boldsymbol{v}_{r}$, respectively.

$$
\begin{aligned}
& \boldsymbol{v}_{t}=\left[\frac{T_{z} x-T_{x} f}{Z}, \frac{T_{z} y}{Z}\right]^{\mathrm{T}} \\
& \boldsymbol{v}_{r}=\left[-\omega_{y} f-\frac{\omega_{y} x^{2}}{f},-\frac{\omega_{y} x y}{f}\right]^{\mathrm{T}}
\end{aligned}
$$

With a pure translational motion $\left(T_{x}\right.$ and $\left.T_{z}\right)$, all vectors point either inwards or outwards with respect to the FOE, $\boldsymbol{p}_{0}$ in Eq. (9). Fig. 2a shows the motion field of a pure translational motion $\left(T_{x}\right.$ and $\left.T_{z}\right)$.

$\boldsymbol{p}_{0}=\left[f \frac{T_{x}}{T_{z}}, 0\right]^{\mathrm{T}}$

If the rotational effect in the $Y$-axis (as shown in Fig. 2b) is eliminated, all the joining lines of the correct matches point either inwards or outwards with respect to the FOE. The proposed algorithm uses this property to reject outliers by eliminating the rotational effect and estimating the location of the FOE. Outliers are identified by using the direction of the joining lines with respect to the FOE. The proposed algorithm is outlined as follows:

\section{Proposed algorithm}

(1) Begin with a set of initial matches between two images.

(2) Eliminate the $Y$-axis rotational effect of the matches with various angles.

(3) Accumulate the joining lines of the matches for each angle.

(4) Save the peak value and its location for each angle.

(5) Choose the maximum peak location as the FOE.

(6) Reject the outliers using the estimated FOE. 
a

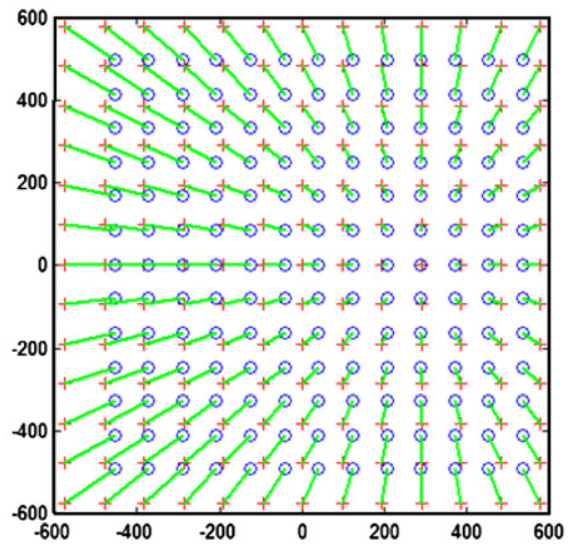

b

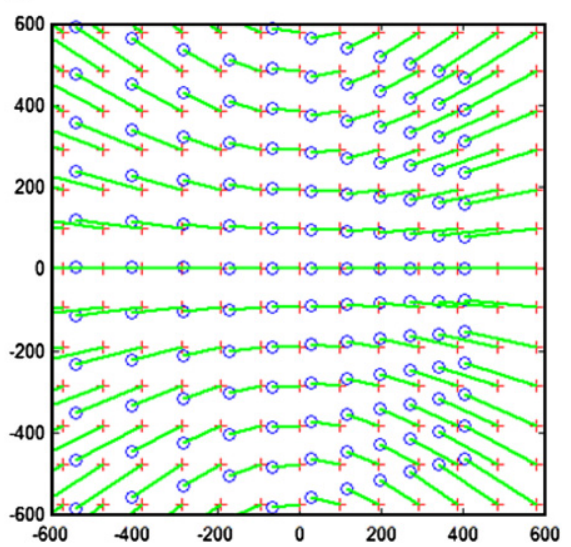

Fig. 2. (a) Motion field of a pure translation $\left(T_{x}\right.$ and $\left.T_{z}\right)$. (b) Motion field of a pure rotation $\left(R_{y}\right)$. The crosses and circles represent the feature points in the first and the second images, respectively, and the lines join the matches.

\section{Estimation of the focus of expansion and outlier rejection}

\subsection{Elimination of the rotational effect}

To estimate the FOE, it is necessary to eliminate the rotational effect. The proposed algorithm uses a homography for this task. The relationship between two images with the same camera center can be expressed by a homography (Hartley and Zisserman, 2000), and the rotational effect between these two images can also be eliminated by a homography. If two cameras are related by a pure rotation, and if camera 1 is a reference camera and camera 2 is the rotated camera (using a $3 \times 3$ rotation matrix $(R)$ ), then the camera matrices of the two cameras $\left(P_{1}\right.$ and $\left.P_{2}\right)$ are written as:

$P_{1}=K[I \mid 0]$

$P_{2}=K[R \mid 0]$
$P_{1}$ and $P_{2}$ are the $3 \times 4$ camera matrices of camera 1 and camera 2, respectively. $K$ is the $3 \times 3$ camera intrinsic parameters matrix of the two cameras. $I$ is a $3 \times 3$ identity matrix. If $\boldsymbol{x}_{1}$ and $\boldsymbol{x}_{2}$ are the projected points of $\boldsymbol{X}$ when using camera 1 and camera 2, respectively, these points are described as:

$\boldsymbol{x}_{1}=P_{1} \boldsymbol{X}=K[I \mid \boldsymbol{0}] \boldsymbol{X}$

$\boldsymbol{x}_{2}=P_{2} \boldsymbol{X}=K[R \mid \boldsymbol{0}] \boldsymbol{X}$

By manipulating Eq. (11), Eq. (12) is obtained.

$\boldsymbol{x}_{1}=K R^{-1} K^{-1} \boldsymbol{x}_{2}=H \boldsymbol{x}_{2}$

According to Eq. (12), $\boldsymbol{x}_{2}$ can be transformed to $\boldsymbol{x}_{1}$ by using a $3 \times 3$ matrix $H$, which can be calculated by using the intrinsic parameters matrix of a pre-calibrated camera and a rotation matrix.

The homography has two uses. The first use is to make the optical axis of the camera parallel to the surface of the road, as shown in Fig. 3a. This makes the camera motion a
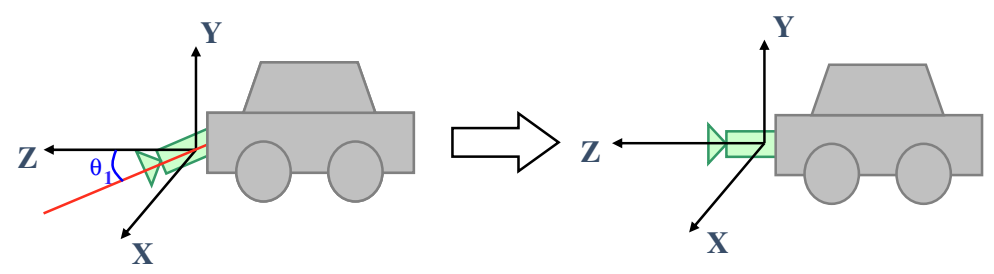

b

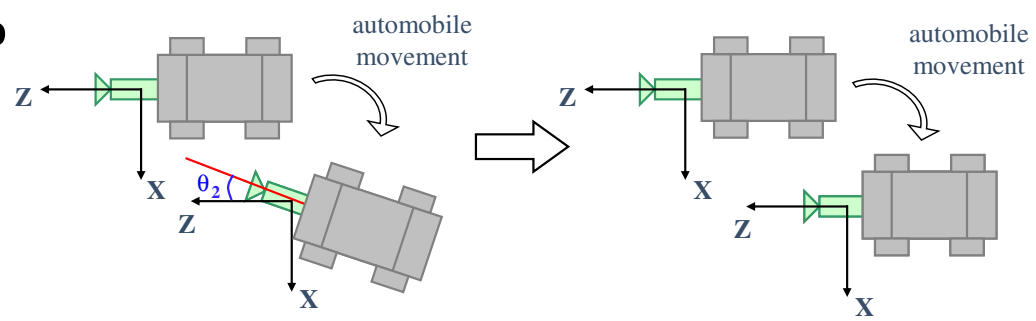

Fig. 3. Two uses of the homography. (a) To make the optical axis parallel to the surface of the road. (b) To eliminate the rotational effect caused by automobile movement. 
consist of two translations $\left(T_{x}\right.$ and $\left.T_{z}\right)$ and one rotation $\left(R_{y}\right)$. The angle $\left(\theta_{1}\right)$ between the optical axis of camera and the surface of the road can be pre-calibrated. The second use is to eliminate the rotational effect caused by automobile movement, as shown in Fig. 3b. This makes the two images related by a pure translation. The angle $\left(\theta_{2}\right)$ caused by automobile movement can not be pre-calibrated so we propose a simple and effective way for estimating the correct rotation angle and the location of the FOE at the same time.

\subsection{Estimation of the focus of expansion}

The $Y$-axis rotation angle and the location of the FOE are estimated by using a homography and an accumulation of joining lines. The accumulation of joining lines is supposed to contain the largest peak value at the location of the FOE, if the feature points in one image are transformed by a homography with the correct rotation angle. This is because all the joining lines of the correct matches pass through the FOE when the rotational effect between the two images is totally eliminated. We estimate the FOE and the $Y$-axis rotation angle by using three steps. First, the feature points in one image are transformed by a homography with a certain rotation angle and their joining lines are accumulated. Second, the peak value and its location in the accumulation are saved. These two steps are repeated with various rotation angles within the search range. Last, the location of the largest peak value and the corresponding angle are recognized as the FOE and the $Y$-axis rotation angle, respectively. Fig. 4 shows an example of the accumulation results. It is shown that the accumulation at $-7^{\circ}$ contains the largest peak value. To find a reliable peak location, the accumulations are smoothed by a $5 \times 5$ averaging filter. Fig. 5 shows how the peak value changes for each rotation angle. The largest peak value is found at $-7^{\circ}$ and this angle is recognized as the $Y$-axis rotation angle of the automobile. Fig. 6 shows the top view of the accumulation with the largest peak value (the boxed accumulation in Fig. 4). The peak location of this accumulation is recognized as the location of the FOE.

\subsection{Outlier rejection}

Once the location of the FOE is estimated, outliers are identified by using two properties. The first property is that all the joining lines of the correct matches pass through the FOE. The second property is that all the joining lines of the correct matches point either inwards or all outwards with respect to the FOE. Using these two properties, outliers are rejected in two steps. In the first step, we measure the angle between two lines. One line is between the FOE and the feature point in the first image and the other line is between the FOE and the corresponding feature point in the second image. This angle has a zero value when the joining line passes exactly through the FOE, so we reject the matches whose angles are larger than a pre-determined threshold angle $\left(\theta_{t}\right)$. This procedure is described in Fig. 7a. In this figure, there are three matches (match 1, 2 , and 3). The circles and crosses indicate the feature points in the first and second images, respectively. Match 1 and match 2 are identified as inliers because $\theta_{1}$ and $\theta_{2}$ are not larger than $\theta_{t}$, but match 3 is identified as an outlier because the $\theta_{3}$ is larger than $\theta_{t}$.

In the second step, we reject the matches that do not follow the common direction. The common direction is either inward or outward with respect to the FOE and it depends

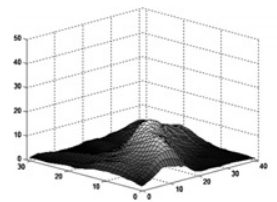

angle $=-15$
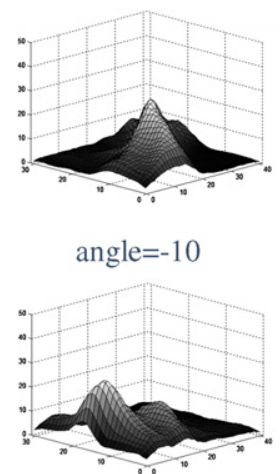

angle $=-5$

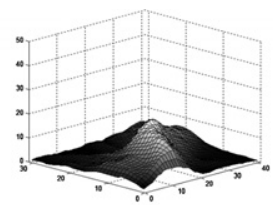

angle $=-14$

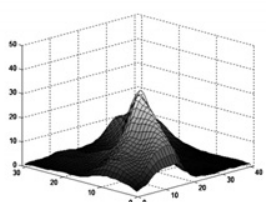

angle $=-9$

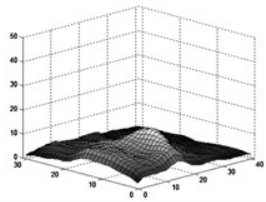

angle $=-4$

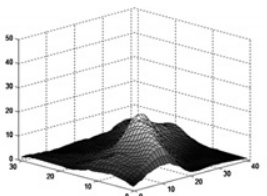

angle $=-13$

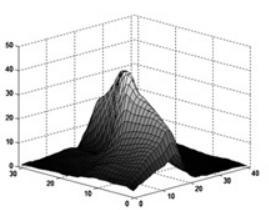

angle $=-8$

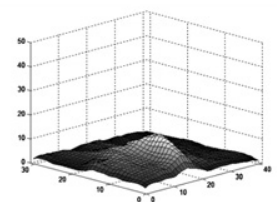

angle $=-3$

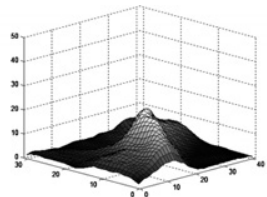

angle $=-12$

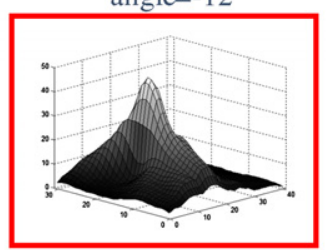

angle $=-7$

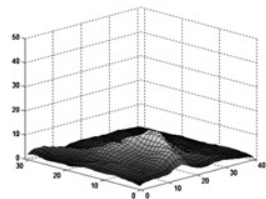

angle $=-2$

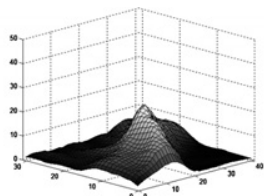

angle $=-11$
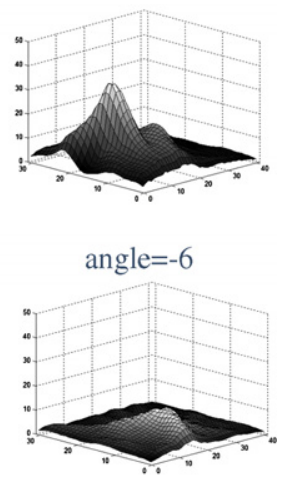

angle $=-1$

Fig. 4. Accumulations of joining lines for different rotation angles. The largest peak appears at angle $=-7$. 


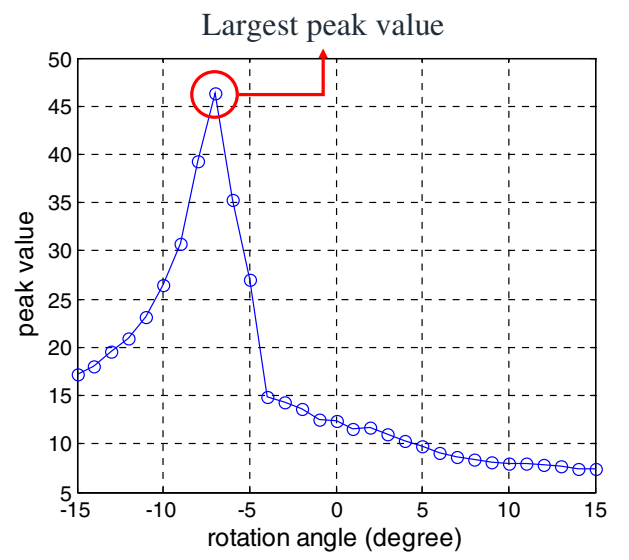

Fig. 5. Changes of the peak value for each rotation angle.

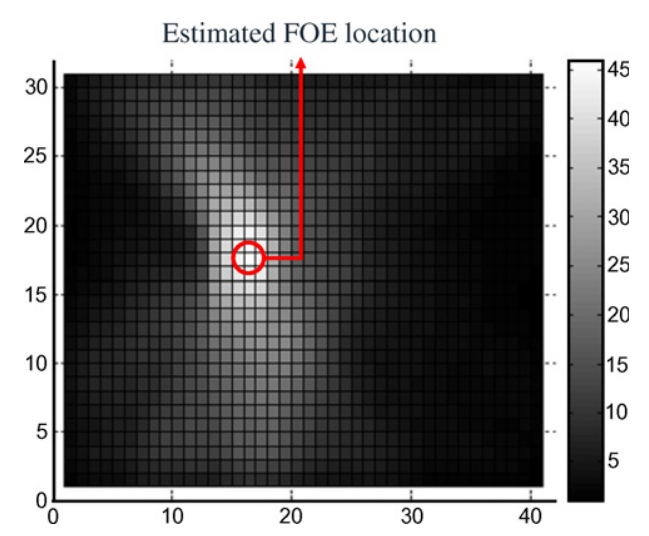

Fig. 6. Estimated location of the FOE in the accumulation.

on the motion of the automobile. This direction is estimated using the retained matches after the first step. We classify the joining line directions (inward or outward) and then use the majority direction as a common direction. This approach is acceptable because most of the outliers are eliminated in the first step. This procedure is described in Fig. 7b. In this figure, the common direction is assumed to be inward. Match 2 is identified as an outlier because it does not follow the common direction.

\section{Experimental results and comparisons}

The proposed algorithm was tested with both synthetic and real image pairs. The real image pairs were acquired with an automobile rear view fisheye camera so that the distortion of the initial matches was removed before they were used. The experiment was divided into four parts. In the first part, we tested the robustness of the proposed algorithm to the $X$ and $Z$-axis rotation (pitching and rolling). In the second part, the performance of the proposed algorithm was tested with various outlier percentages $(10-90 \%)$. In the third part, we compared the performance of the proposed algorithm with Adam's algorithm (Adam et al., 2001). In the last part, we used the proposed algorithm as a pre-processing step of RANSAC to demonstrate significant reductions of computational cost.

Two criteria were used to assess performance. One is the rejected inlier percentage (false negative rate) and the other is the retained outlier percentage (false positive rate) (Fawcett, 2004). For both criteria, a lower percentage means better performance. A good outlier rejection algorithm should have low percentages for both criteria.

\subsection{Simulation of automobile motion}

The following steps describe the process we used to design a simulation environment for automobile motion.

(1) Set up a synthetic camera with a focal length and field of view of $2 \mathrm{~mm}$ and $160^{\circ}$, respectively.

(2) Make 200-500 3D points (depth range: $5-10 \mathrm{~m}$ ) with locations within the field of view.

(3) Set a distance interval $(d=1.4 \mathrm{~m})$ between two camera positions and define the minimum turning radius $\left(r_{\min }=5.5 \mathrm{~m}\right)$ of the automobile. $(1.4 \mathrm{~m}$ refers to the distance that an automobile can move for $1 \mathrm{~s}$ at $5 \mathrm{~km} / \mathrm{h})$.

(4) Calculate the maximum steering angle $\left(R_{y_{\_} \max }=15^{\circ}\right)$ using Eq. (13) and randomly select a steering angle $\left(R_{y}\right)$ within the range $\left(-R_{y \_ \text {max }} \sim+R_{y \_ \text {max }}\right)$.

(5) Calculate the turning radius $(r)$, the $X$-axis translation $\left(T_{x}\right)$, and the $Z$-axis translation $\left(T_{z}\right)$ using Eq. (14).

(6) Project the 3D points on the two cameras.
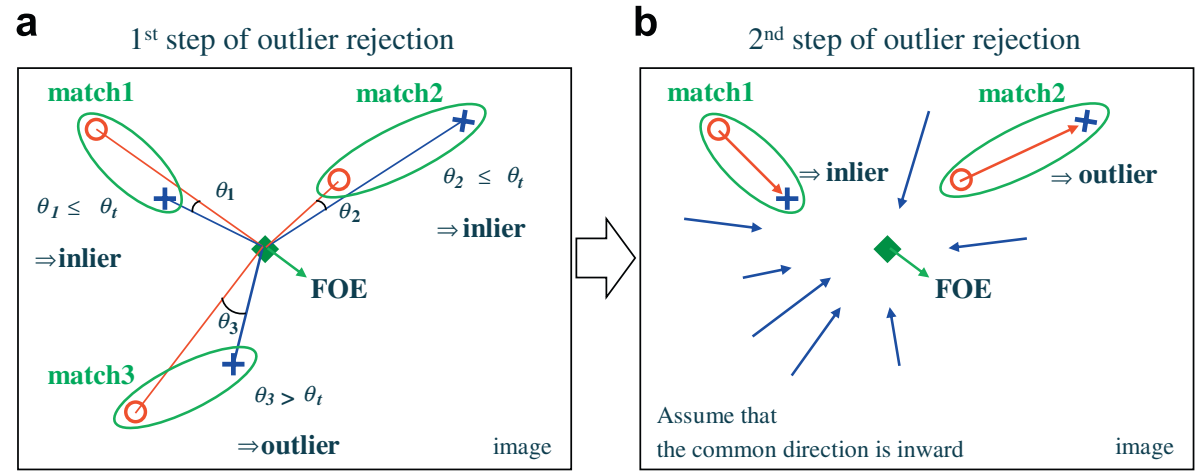

Fig. 7. Two steps of outlier rejection. The circles and crosses indicate the feature points in the first and second images, respectively. 
$R_{y_{-} \max }=d / r_{\min }$

$r=d / R_{y}$

$T_{x}=r-r \cos \left(R_{y}\right)$

$T_{z}=r \tan \left(R_{y}\right)$

* The $Y$-axis translation $\left(T_{y}\right)$ is usually small in automobile motion and it does not disturb matches to make the FOE, so we simply let the $Y$-axis translation $\left(T_{y}\right)$ vary from $-0.1 \mathrm{~m}$ to $+0.1 \mathrm{~m}$.

\subsection{Robustness of the proposed algorithm}

Since automobile motion may not be ideal in practical situations, we tested the robustness of the proposed algorithm in terms of the $X$ and $Z$-axis rotation (pitching and rolling) with 1050 synthetic image pairs. The search range of the $Y$-axis rotation angle was set from $-15^{\circ}$ to $+15^{\circ}$ at intervals of $1^{\circ}$. The accumulation bin size and the threshold angle $\left(\theta_{t}\right)$ were empirically set as $20 \times 20$ pixels and $3^{\circ}$. Under these circumstances, we contaminated the initial matches by $50-90 \%$ of outliers while changing the $X$ and $Z$-axis rotation angles from $0^{\circ}$ to $10^{\circ}$. The test results for these initial matches are shown in Fig. $8 \mathrm{a}$ and b. This figure shows that the proposed algorithm is robust to the $X$-axis rotation but less robust to the $Z$-axis rotation. The robustness to the $X$-axis rotation is because this rotation approximately moved the FOE in a vertical direction and slightly disturbed the correct matches to make the FOE. A small disturbance was offset by a sufficiently large accumulation bin size. The sensitivity to the $Z$-axis rotation is because this rotation largely disturbed the correct matches to make the FOE. However, as the $Z$-axis translation $\left(T_{z}\right)$ is dominant in automobile motion, many correct matches tend to point to a common region despite a small disturbance of the $Z$-axis rotation.

To show the robustness of the proposed algorithm more clearly, we depict Fig. 8 as Fig. 9, using the outlier percentage ratio. This figure shows the ratio between the initial
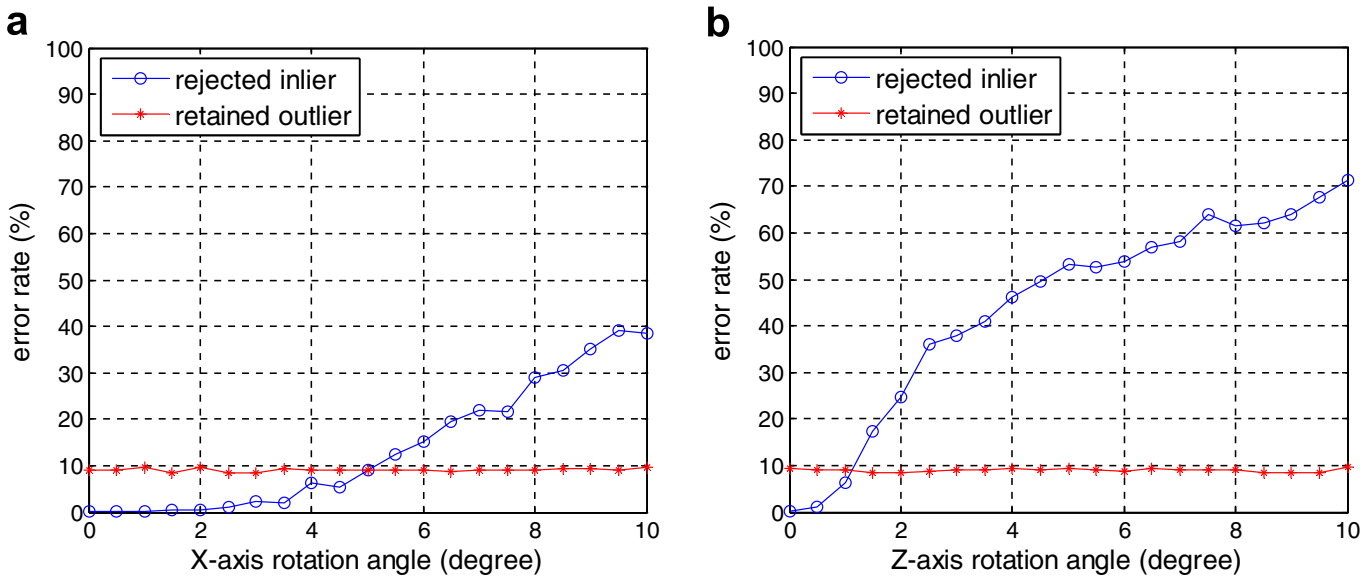

Fig. 8. Robustness of the proposed algorithm to (a) the $X$-axis rotation (pitching) and (b) the $Z$-axis rotation (rolling).
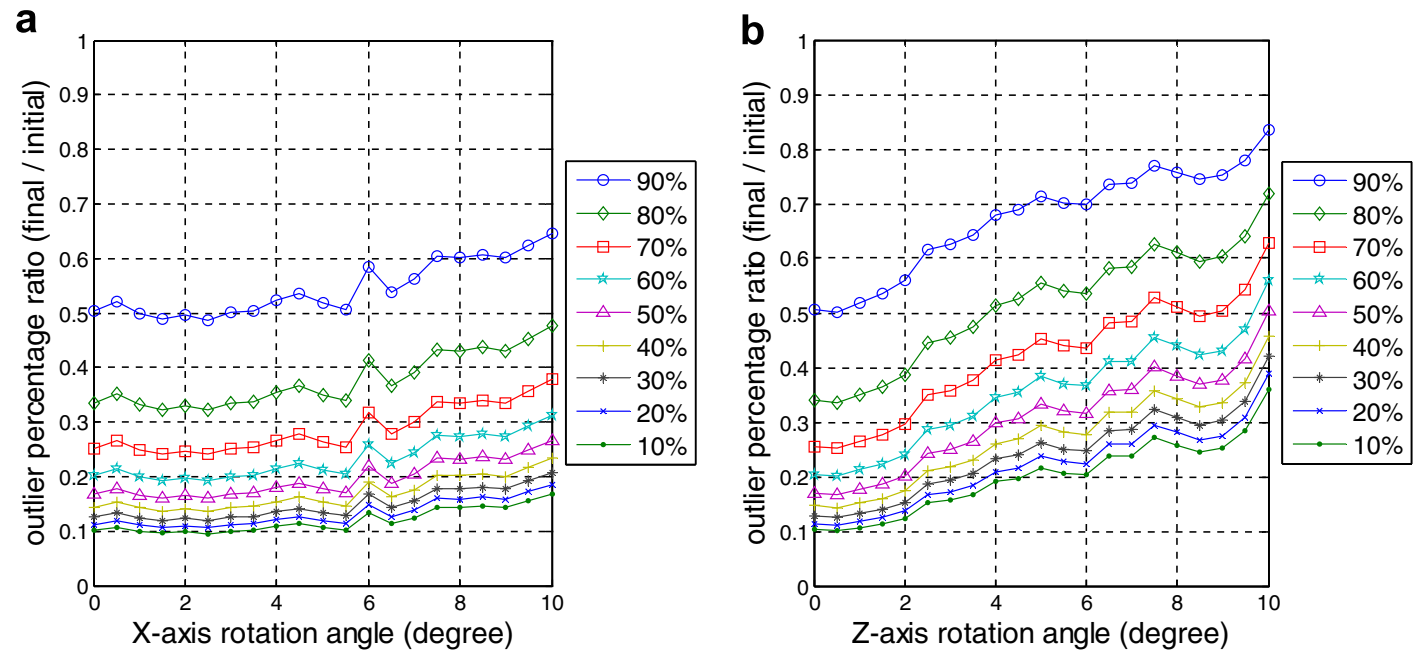

Fig. 9. The ratio between the initial outlier percentage and the final outlier percentage with respect to (a) the $X$-axis rotation angle, and (b) the $Z$-axis rotation angle. Lines in each graph correspond to the initial outlier percentages. 


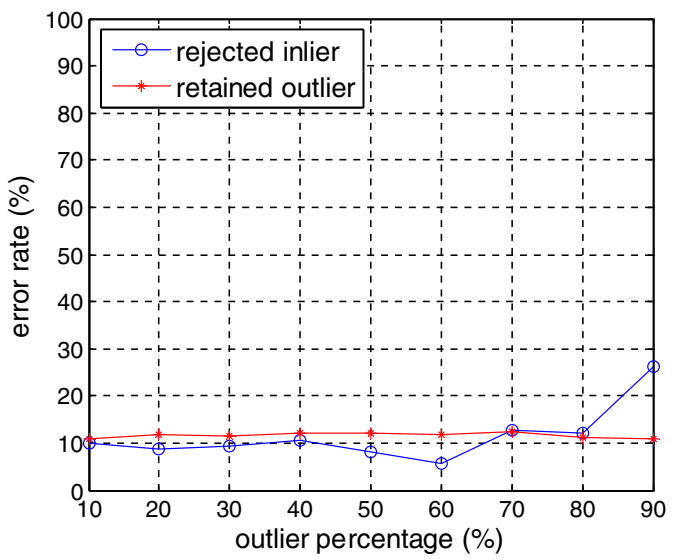

Fig. 10. Performance of the proposed algorithm when using 450 synthetic image pairs.
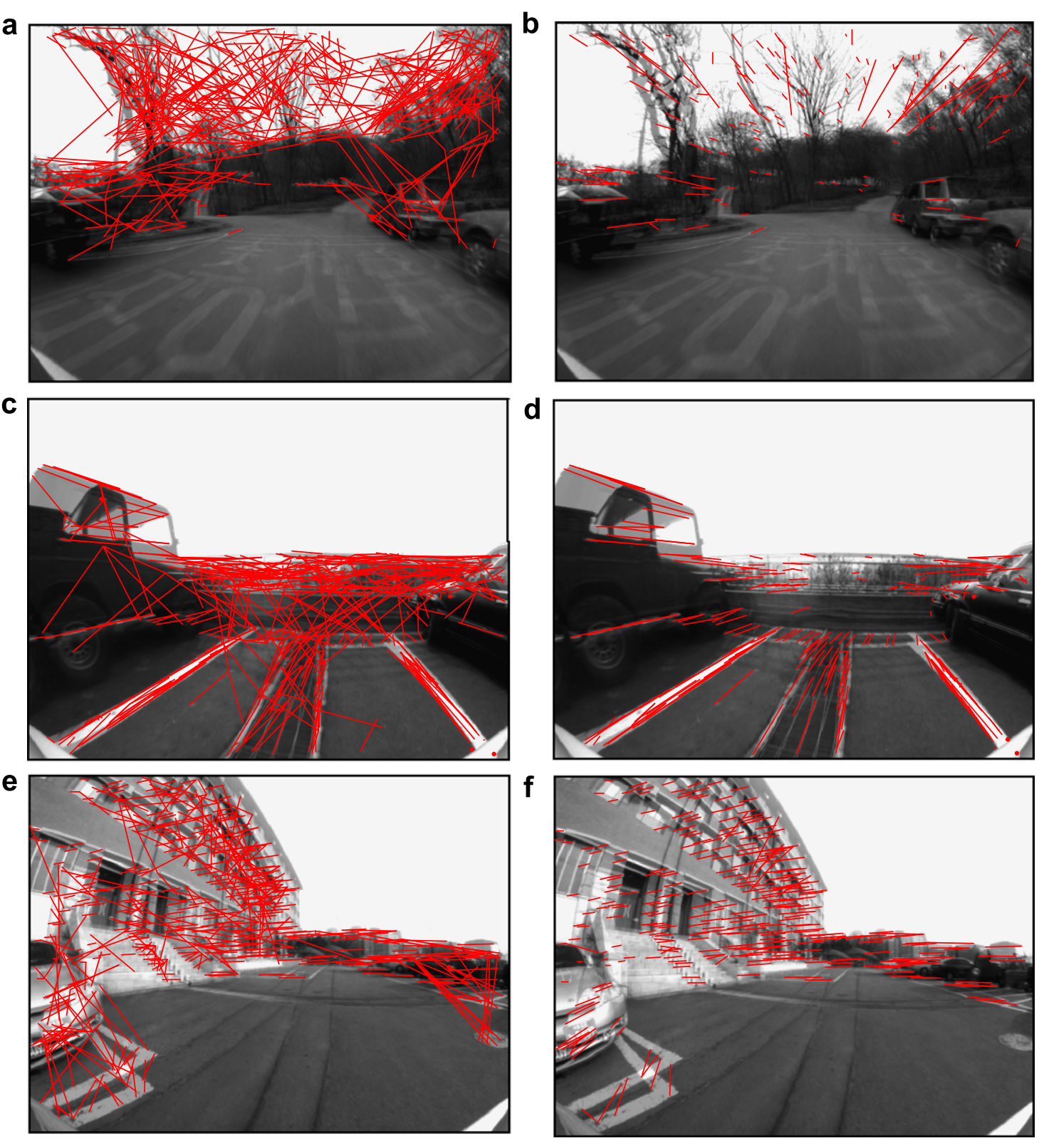

Fig. 11. Three examples of outlier rejection results. (a), (c), and (e) are the initial matches of image pair number 3, 6, and 9. (b), (d), and (f) are their outlier rejection results.

outlier percentage and the final outlier percentage. A lower outlier percentage ratio means that the algorithm performs well. According to Fig. 9a, when the initial matches included fewer than $80 \%$ of the outliers, the proposed algorithm reduced the outlier percentage by less than half until the $X$-axis rotation was smaller than $10^{\circ}$. According to Fig. 9b, when the initial matches included fewer than $70 \%$ of the outliers, the proposed algorithm reduced the outlier percentage by less than half until the $Z$-axis rotation was smaller than $7^{\circ}$. The proposed algorithm is robust to $X$-axis rotation but less robust to $Z$-axis rotation and it fails when automobile motion includes large $X$ and $Z$-axis rotations. However, in practical automobile motion, there is usually a small $X$-axis rotation and the $Z$-axis rotation rarely occurs.

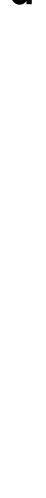

$f$ 


\subsection{Performance of the proposed algorithm}

The proposed algorithm was tested with 450 synthetic image pairs and 10 real image pairs. First, we present the

Table 2

Performance of the proposed algorithm for 10 real image pairs

\begin{tabular}{|c|c|c|c|c|c|c|}
\hline $\begin{array}{l}\text { Image } \\
\text { pair } \\
\text { no. }\end{array}$ & $\begin{array}{l}\text { Total } \\
\text { matches }\end{array}$ & Inliers & Outliers & $\begin{array}{l}\text { Outlier } \\
\text { percentage } \\
(\%)\end{array}$ & $\begin{array}{l}\text { Retained } \\
\text { outlier } \\
\text { percentage } \\
(\%)\end{array}$ & $\begin{array}{l}\text { Rejected } \\
\text { inlier } \\
\text { percentage } \\
(\%)\end{array}$ \\
\hline 1 & 494 & 60 & 434 & 87.9 & 11.3 & 1.7 \\
\hline 2 & 454 & 52 & 402 & 88.5 & 9.0 & 3.8 \\
\hline 3 & 535 & 105 & 430 & 80.4 & 11.6 & 0.0 \\
\hline 4 & 517 & 116 & 401 & 77.6 & 7.7 & 0.9 \\
\hline 5 & 507 & 141 & 366 & 72.2 & 11.5 & 9.9 \\
\hline 6 & 518 & 142 & 376 & 72.6 & 21.5 & 6.3 \\
\hline 7 & 508 & 173 & 335 & 65.9 & 15.8 & 7.5 \\
\hline 8 & 531 & 231 & 300 & 56.5 & 23.3 & 3.9 \\
\hline 9 & 522 & 305 & 217 & 41.6 & 10.6 & 0.7 \\
\hline 10 & 540 & 255 & 285 & 52.8 & 12.3 & 2.7 \\
\hline
\end{tabular}

Table 3

Comparison of computation time between RANSAC and RANSAC with the proposed algorithm for real image pairs

\begin{tabular}{llllll}
\hline $\begin{array}{l}\text { Image } \\
\text { pair no. }\end{array}$ & $\begin{array}{l}\text { Outlier } \\
\text { percentage } \\
(\%)\end{array}$ & \multicolumn{4}{l}{ Computation time $(\mathrm{s})$} \\
\cline { 3 - 6 } & & & RANSAC & \multicolumn{2}{l}{ Proposed algorithm + RANSAC } \\
\cline { 3 - 6 } & & & $\begin{array}{l}\text { Proposed } \\
\text { algorithm }\end{array}$ & RANSAC & Total \\
\hline 1 & 87.9 & 9729.937 & 0.147 & 0.547 & 0.694 \\
2 & 88.5 & 19014.688 & 0.133 & 0.150 & 0.283 \\
3 & 80.4 & 666.235 & 0.164 & 0.084 & 0.248 \\
4 & 77.6 & 955.078 & 0.141 & 0.092 & 0.233 \\
5 & 72.2 & 223.906 & 0.148 & 0.097 & 0.245 \\
6 & 72.6 & 35.000 & 0.156 & 0.183 & 0.339 \\
7 & 65.9 & 13.563 & 0.156 & 0.055 & 0.211 \\
8 & 56.5 & 5.969 & 0.161 & 0.075 & 0.236 \\
9 & 41.6 & 1.078 & 0.153 & 0.036 & 0.189 \\
10 & 52.8 & 1.906 & 0.164 & 0.039 & 0.203 \\
\hline
\end{tabular}

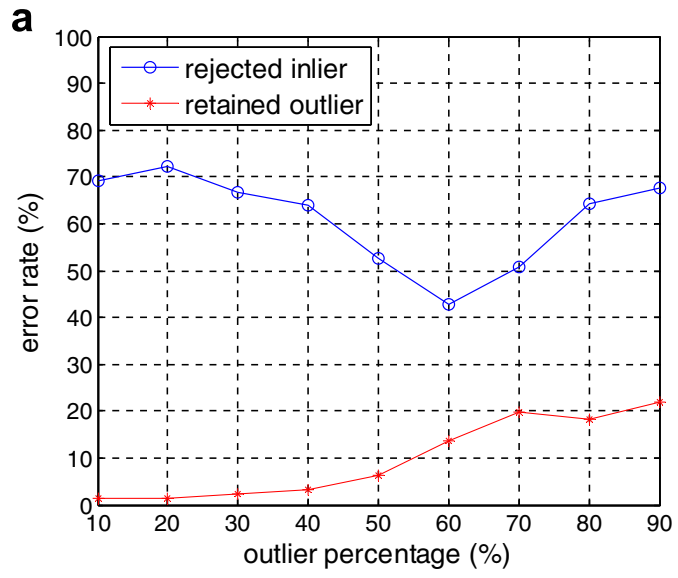

results for the synthetic image pairs, and then we present the results for the real image pairs. Each synthetic image pair was generated in the same way described in Section 4.2. The only differences were that the $X$ and $Z$-axis rotation angles varied between $-5^{\circ} \sim+5^{\circ}$ and $-2^{\circ} \sim+2^{\circ}$, respectively. This is because the $Z$-axis rotation occurs less frequently than the $X$-axis rotation in a real automobile motion. The rejected inlier percentage and the retained outlier percentage for the synthetic image pairs are shown in Fig. 10. The proposed algorithm rejected $5.6-12.1 \%$ of the inliers until the outlier percentage was less than $80 \%$ but it rejected $26 \%$ of the inliers when the outlier percentage was $90 \%$. This may have been because $10 \%$ of inliers are insufficient to produce a peak value at the FOE when there are large disturbances caused by the $X$ and $Z$-axis rotations. The retained outlier percentages were almost constant in the range of $10.8-12.4 \%$ because outliers were uniformly distributed all over the image so the fixed threshold angle $\left(\theta_{t}\right)$ included almost the same percentage of outliers.

The real image pairs were acquired with an automobile rear view fisheye camera with horizontal and vertical fields of view of about $154^{\circ}$ and $115^{\circ}$, respectively. The image resolution of the camera was $1024 \times 768$ pixels. The Harris corner detector (Harris and Stephens, 1988) and the $7 \times 7$ SSD (Sum of Squared Distance) matcher were used to find initial matches. Fig. 11 shows three examples of initial matches on the real image pairs and their outlier rejection results. The image pair numbers in Fig. 11 correspond to the image pair numbers in Tables 2 and 3 . Table 2 shows the performance of the proposed algorithm on 10 real image pairs. The ground truth data were manually designated. The proposed algorithm rejected $86.5 \%$ of outliers and retained $96.3 \%$ of inliers. These are the average percentages obtained with 10 image pairs. The most severe case was found in image pair number 2 whose initial matches included $88.5 \%$ of outliers. Even in this case, the proposed algorithm successfully rejected $91.0 \%$ of the outliers while retaining $96.2 \%$ of the inliers. The reason that

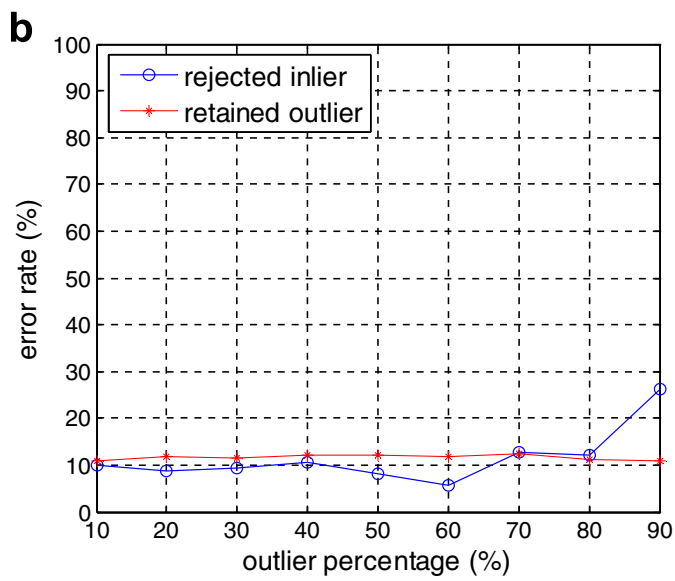

Fig. 12. Performance comparison between Adam's algorithm and the proposed algorithm for synthetic image pairs. (a) Error rates of Adam's algorithm (b) error rates of the proposed algorithm. 
the retained inlier percentages in the real image pairs were higher than those in the synthetic image pairs is that the $X$ and $Z$-axis rotations are usually small in real situations.

\subsection{Comparison between Adam's algorithm and the proposed algorithm}

We compared the proposed algorithm with Adam's algorithm (Adam et al., 2001). As mentioned in their paper, when working with this algorithm, image pairs have to be taken by a narrow-angle camera. This limitation is critical because intelligent vehicle applications usually require wide-angle cameras for observing rear views. For this reason, it was assumed that Adam's algorithm would fail when the scene points were from wide viewing angles. We applied Adam's algorithm to synthetic and real image pairs using its original code available on the website (Adam, 2007). The comparison results for 450 synthetic image pairs are shown in Fig. 12. Adam's algorithm rejected a large portion of inliers $(42.9-72.2 \%)$, even though it retained a small portion of outliers $(1.3-22.0 \%)$. All the matches in the wide-angle of view were identified as outliers because these matches could not contribute to making a main orientation so they did not follow it. Fig. 13 shows the outlier rejection results of Adam's algorithm and the proposed algorithm when using real image pairs. Adam's algorithm worked only within a small region and rejected all the matches outside that region. However, the proposed
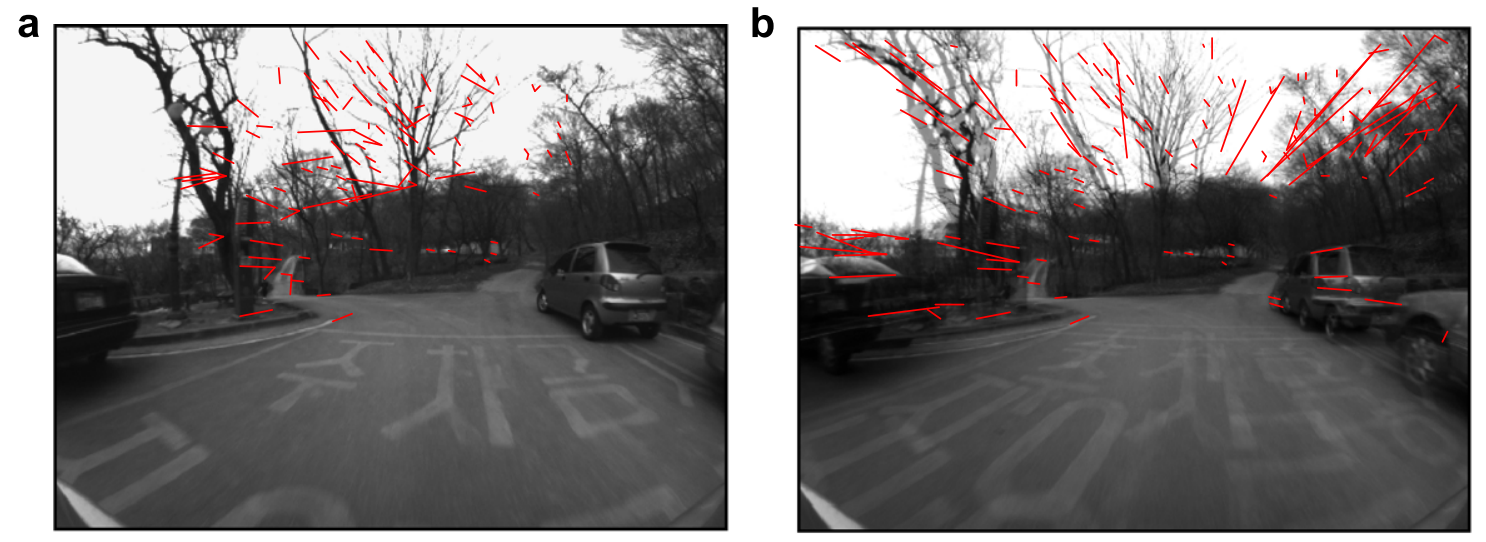

C

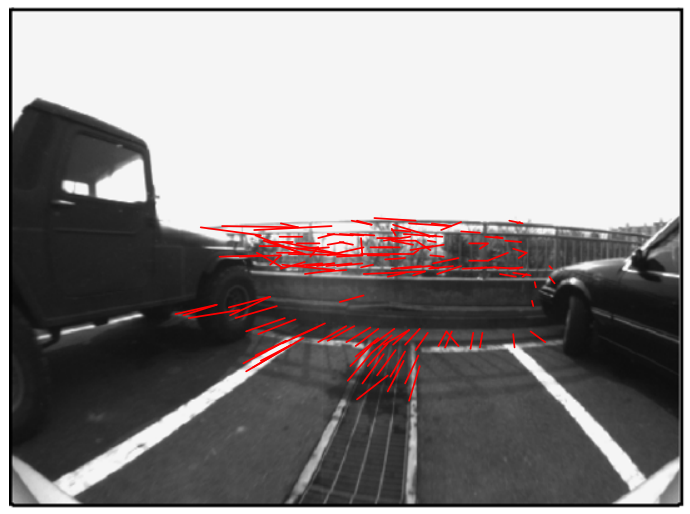

e

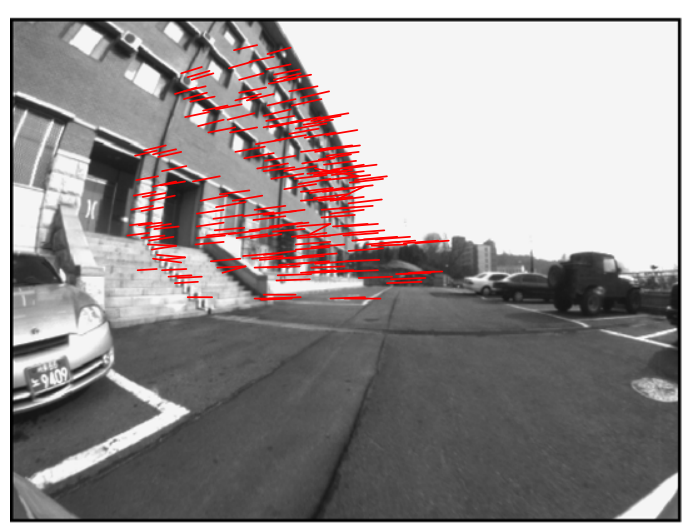

d

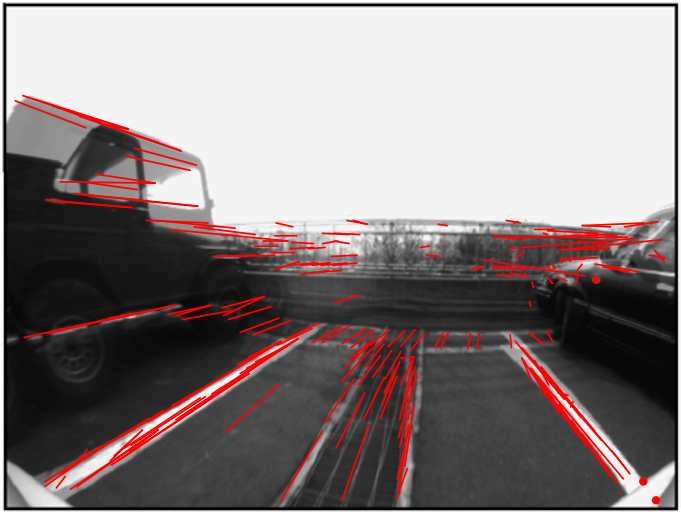

f

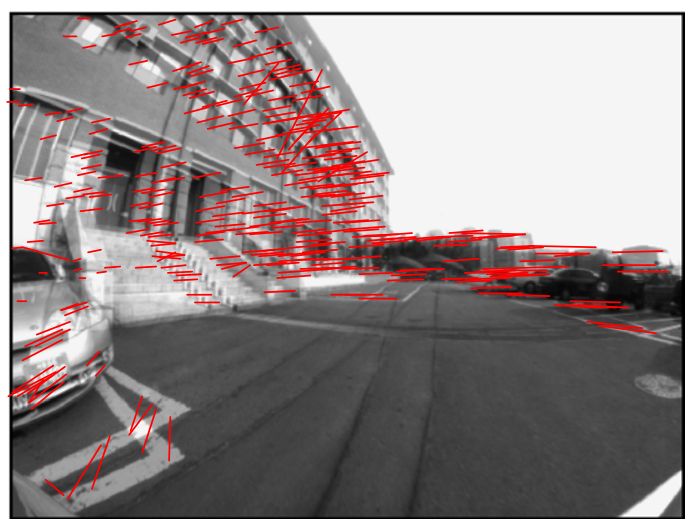

Fig. 13. Comparison of outlier rejection results between Adam's algorithm and the proposed algorithm. (a), (c), and (e) are the results from Adam's algorithm for image pairs number 3,6, and 9. (b), (d), and (f) are the results from the proposed algorithm for the same image pairs. 
algorithm successfully worked in both narrow and wide viewing angles.

Fig. 14 shows the rejected inlier percentages and the retained outlier percentages of Adam's algorithm and the proposed algorithm for 10 real image pairs. The retained outlier percentage and the rejected inlier percentage of Adam's algorithm were $11.8 \%$ and $34.6 \%$, respectively, and those of the proposed algorithm were $13.5 \%$ and $3.7 \%$, respectively. These are the average percentages for the 10 image pairs. The retained outlier percentage of Adam's algorithm was slightly smaller than that of the proposed algorithm because it rejected all the inliers and outliers in the wide viewing angle. With the same initial matches, the proposed algorithm rejected almost the same percentage of outliers (only $1.7 \%$ difference) and retained $30.9 \%$ more inliers than Adam's algorithm.

\subsection{Comparison between RANSAC and RANSAC with the proposed algorithm}

As mentioned in the introduction, the proposed algorithm can be used with a robust estimation algorithm (such as RANSAC) to lower computational costs. To show the efficiency of the proposed algorithm as a pre-processing step of a robust estimation algorithm, we compared the computation time of RANSAC alone and RANSAC with the proposed algorithm using 450 synthetic image pairs and 10 real image pairs. For a trustable comparison, we used Peter's RANSAC code available on the website (Peter, 2007). These algorithms were run in MATLAB using a $1.73 \mathrm{GHz}$ Intel Pentium M processor.

The comparison results for the synthetic image pairs are shown in Fig. 15. Fig. $15 \mathrm{~b}$ is an enlarged version of Fig. 15a. The computation time of RANSAC dramatically increased with respect to outlier percentage (computation time with $90 \%$ of outliers was over $24 \mathrm{~h}$ so we could not measure it). However, the computation time of the proposed algorithm was independent of the outlier percentage so it significantly reduced the computation time of RANSAC. With $90 \%$ of outliers, the computation time of RANSAC with the proposed algorithm was a little bit higher than the others. This is because the performance of the proposed algorithm was slightly degraded with $90 \%$ of outliers, as shown in Fig. 10. In Fig. 15b, it can be seen that
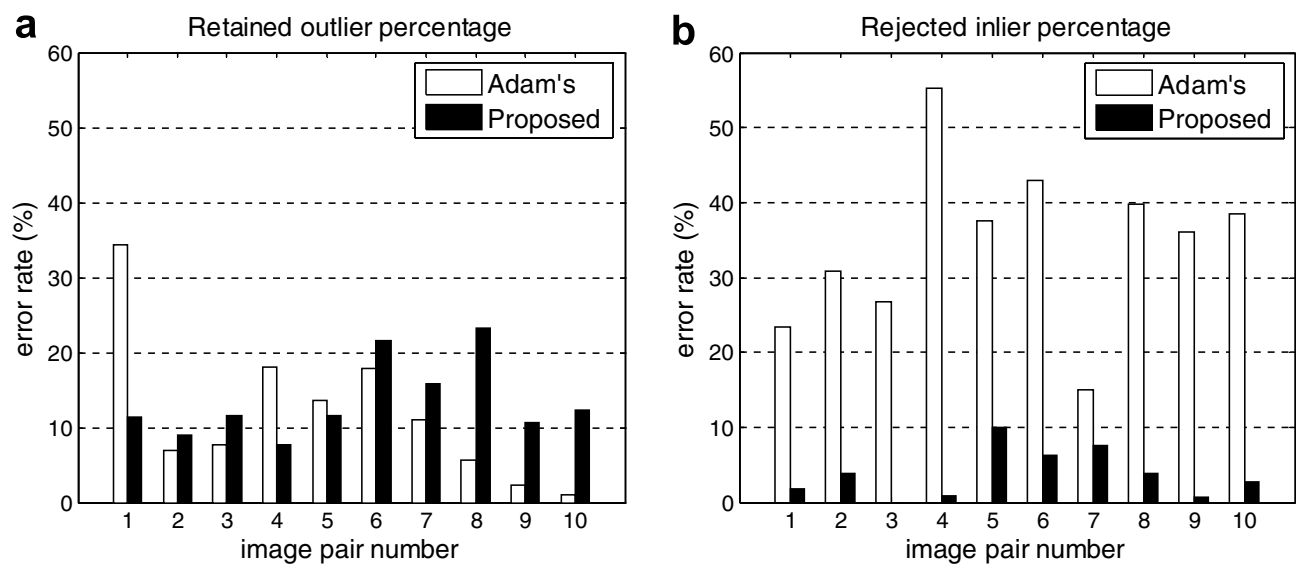

Fig. 14. Performance comparison between Adam's algorithm and the proposed algorithm for 10 real image pairs (a) retained outlier percentages (b) rejected inlier percentages.
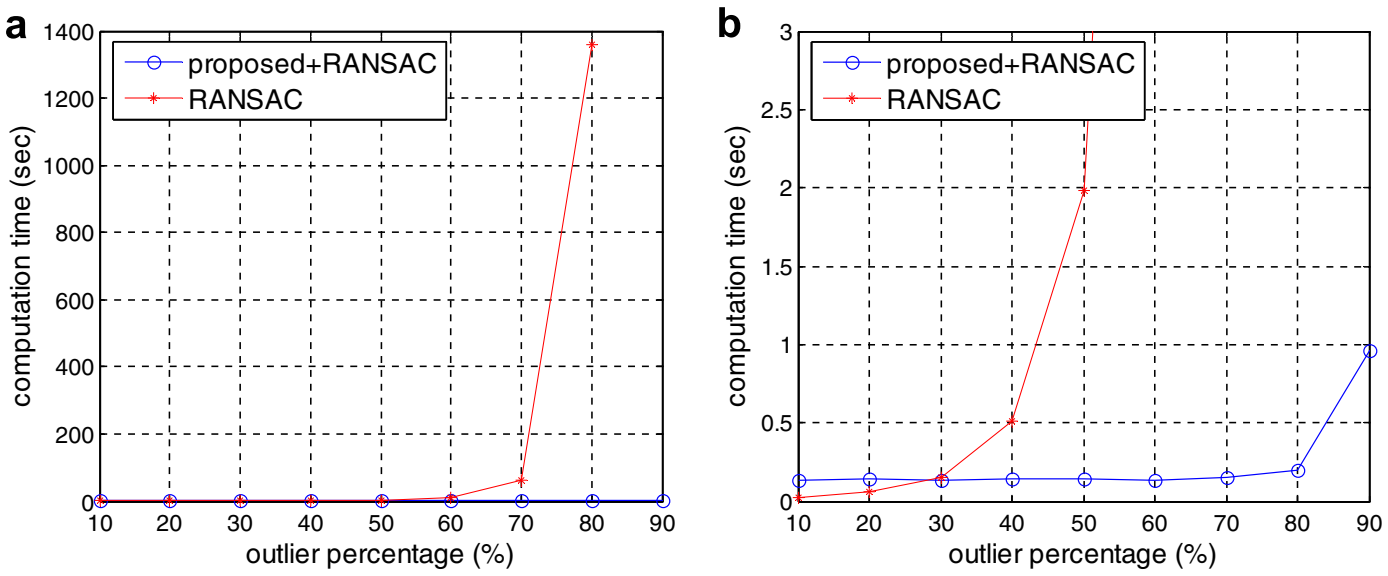

Fig. 15. Comparison of computation time between RANSAC and RANSAC with the proposed algorithm for synthetic image pairs (b) is an enlarged version of (a). 
RANSAC alone was faster than RANSAC with the proposed algorithm when the outlier percentage was lower than $28 \%$. However, the proposed algorithm made RANSAC more effective and practical when the outlier percentage was higher than $28 \%$.

Table 3 shows the computation time comparison for 10 real image pairs. When the outlier percentages of the real image pairs varied from $41.6 \%$ to $88.5 \%$, the computation times of RANSAC varied from $1.078 \mathrm{~s}$ to $19014.688 \mathrm{~s}$ and the computation times of RANSAC with the proposed algorithm varied from only 0.189$0.694 \mathrm{~s}$. These results show that the proposed algorithm significantly reduced the computation time of RANSAC when the image pairs were contaminated by a large percentage of outliers. Parameter estimation accuracy of RANSAC depends on the error tolerance used to determine the consensus set (Fischler and Bolles, 1981). Since RANSAC and RANSAC with the proposed algorithm used the same error tolerance, their accuracies were almost identical.

\section{Conclusions}

This paper proposes a new algorithm for rejecting outliers in image pairs acquired with an automobile rear view camera. Outliers can affect the performances of motion estimation and 3D reconstruction, which are widely used techniques in intelligent vehicle applications. The proposed algorithm estimates the focus of expansion (FOE) and rejects outliers using the properties of the FOE. The proposed algorithm offers four advantages. (1) Complexity is independent of the outlier percentage. (2) It works when the outlier percentage is large $(80-90 \%)$. (3) It is simple and fast. (4) It can be used as a pre-processing step of a robust estimation algorithm. Experimental results showed that the proposed algorithm rejected $86.5 \%$ of outliers while retaining $96.3 \%$ of inliers and it took only $0.152 \mathrm{~s}$ with a $1.73 \mathrm{GHz}$ Intel Pentium $\mathrm{M}$ processor. These are the average percentages for 10 real image pairs. As a preprocessing step of RANSAC, this algorithm significantly reduced computation time. This makes RANSAC practical when initial matches are contaminated by a large percentage of outliers. In the future, we will make efforts to improve the robustness of the algorithm against the pitching and rolling.

\section{Acknowledgement}

This work was supported by the Korea Science and Engineering Foundation (KOSEF) through the Biometrics Engineering Research Center (BERC) at Yonsei University.

\section{References}

Adam, A., Rivlin, E., Shimshoni, I., 2001. ROR: Rejection of outliers by rotations. IEEE Trans. Pattern Anal. Machine Intell. 23 (01), 78-84.
Adam, A., 2007. ROR: Rejection of outliers by rotations. http:// www.cs.technion.ac.il/Labs/Isl/Project/Projects_done/ror/.

Burger, W., Bhanu, B., 1990. Estimating 3-D egomotion from perspective image sequences. IEEE Trans. Pattern Anal. Machine Intell. 12 (11), 1040-1058.

Batavia, P.H., Pomerleau, D.E., Thorpe, C.E., 1997. Overtaking vehicle detection using implicit optical flow. IEEE Conference on Intelligent Transportation Systems, pp. 729-734.

Dufournaud, Y., Schmid, C., Horaud, R., 2000. Matching images with different resolutions. In: Proc. IEEE Conf. Comput. Vision Pattern Recognition, pp. 612-618.

Fawcett, T., 2004. ROC Graphs: Notes and practical considerations for researchers. HP Laboratories, pp. 1-38.

Fintze1, K., Bendahan, R., Vestri, C., Bougnoux, S., 2004. 3D Parking assistant system. Proceedings of the IEEE Intelligent Vehicles Symposium, pp. 881-886.

Fischler, M.A., Bolles, R.C., 1981. Random sample consensus: A paradigm for model fitting with applications to image analysis and automated cartography. Commun. ACM 24 (6), 381-395.

Gandhi, T., Trivedi, M., 2005. Parametric ego-motion estimation for vehicle surround analysis using an omnidirectional camera. Machine Vision Appl. 16 (2), 85-95.

Goshtasby, A.A., 2005. 2-D and 3-D Image Registration for Medical, Remote Sensing, and Industrial Applications. Wiley Publishers.

Grinstead, B., Koschan, A., 2006. Outlier rejection by oriented tracks to aid pose estimation from video. Pattern Recognition Lett. 27 (01), 37-48.

Harris, C., Stephens, M., 1988. A combined corner and edge detector. Proceedings of 4th Alvey Vision Conference, pp. 147-151.

Hartley, R., 1995. In defense of the 8-point algorithm. In: Proc. Internat. Conf. Comput. Vision, pp. 1064-1070.

Hartley, R., Zisserman, A., 2000. Multiple View Geometry in Computer Vision. Cambridge University Press.

Jung, H.G., Kim, D.S., Yoon, P.J., Kim, J., 2006. Parking slot markings recognition for automatic parking assist system. In: Proc. IEEE Intell. Vehicles Symp., pp. 106-113.

Lowe, D., 2004. Distinctive image features from scale-invariant keypoints. Internat. J. Comput. Vision 60 (02), 91-110.

Lucas, B.D., Kanade, T., 1981. An iterative image registration technique with an application to stereo vision. In: Proc. Internat. Joint Conf. Artif. Intell., pp. 674-679.

Ma, Y., Soatto, S., 2000. An Invitation to 3-D Vision. Springer.

Mikolajczyk, K., Schmid, C., 2004. Scale and affine invariant interest point detectors. Internat. J. Comput. Vision 60 (01), 63-86.

Mikolajczyk, K., Schmid, C., 2005. A performance evaluation of local descriptors. IEEE Trans. Pattern Anal. Machine Intell. 27 (10), 1615-1630.

Mouragnon, E., Dekeyser, F., Sayd, P., Lhuillier, M., Dhome, M., 2006a. Real time localization and 3D reconstruction. IEEE Comput. Soc. Conf. Comput. Vision Pattern Recognition, 1, pp. 17-22.

Mouragnon, E., Lhuillier, M., Dhome, M., Dekeyser, F., Sayd, P., $2006 \mathrm{~b}$. Monocular vision based SLAM for mobile robots. In: Proc. 18th Internat. Conf. Pattern Recognition, 3, pp. 20-24.

Peter, K., 2007. MATLAB and Octave functions for computer vision and image processing. http://www.csse.uwa.edu.au/ pk/Research/MatlabFns/.

Royer, E., Lhuillier, M., Dhome, M., Chateau, T., 2005. Localization in urban environments: Monocular vision compared to a differential GPS sensor. In: Proc. IEEE Comput. Soc. Conf. Comput. Vision Pattern Recognition, 2, pp. 114-121.

Salvi, J., Armangue, X., Pages, J., 2001. A survey addressing the fundamental matrix estimation problem. In: Proc. Internat. Conf. Image Process., pp. 209-212.

Schmid, C., Mohr, R., 2000. Evaluation of interest point detectors. Internat. J. Comput. Vision 37 (02), 151-172.

Smith, S., Brady, M., 1997. Susan - a new approach to low level image processing. Internat. J. Comput. Vision 23 (01), 45-78.

Suhr, J.K., Jung, H.G., Bae, G., Kim, J., 2007. Free parking space detection using optical flow-based Euclidean 3D reconstruction. IAPR Conf. Machine Vision Appl., pp. 563-566. 
Sun, Z., Bebis, G., Miller, R., 2006. On-road vehicle detection: A review. IEEE Trans. Pattern Anal. Machine Intell. 28 (5), 694-711.

Takahashi, A., Ninomiya, Y., Ohta, M., Nishida, M., Takayama, M., 2002. Rear view lane detection by wide angle camera. IEEE Intell. Vehicle Symp., pp. 148-153.

Tomasi, C., Shi, J., 1994. Good features to track. In: Proc. IEEE Conf. Comput. Vision Pattern Recognition, pp. 593-600.

Torr, P., Murray, D., 1997. The development and comparison of robust methods for estimating the fundamental matrix. Internat. J. Comput. Vision 24 (03), 271-300.

Torr, P., Zisserman, A., 1998. Robust computation and parameterization of multiple view relations. In: Proc. IEEE Internat. Conf. Comput. Vision, pp. 727-732.
Trucco, E., Verri, A., 1998. Introductory Techniques for 3-D Computer Vision. Prentice Hall.

Vestri, C., Bougnoux, S., Bendahan, R., Fintzel, K., Wybo, S., Abad, F., Kakinami, T., 2005. Evaluation of a vision-based parking assistance system. In: Proc. 8th Internat. IEEE Conf. Intell. Transport. Syst., pp. 56-60.

Yamaguchi, K., Kato, T., Ninomiya, Y., 2006. Moving obstacle detection using monocular vision. IEEE Intell. Vehicles Symp., pp. 288-293.

Zhang, Z., 1998. Determining the epipolar geometry and its uncertainty: A review. Internat. J. Comput. Vision 27 (02), 161-198.

Zhang, W., Kosecka, J., 2006. Generalized RANSAC framework for relaxed correspondence problems. Third International Symposium on 3D Data Processing, Visualization, and Transmission, pp. 854-860. 